\title{
Curvelet Transform Based on Edge Preserving Filter for Retinal Blood Vessel Segmentation
}

\author{
Sonali Dash ${ }^{1}$, Sahil Verma ${ }^{2, *}$, Kavita ${ }^{2}$, N. Z. Jhanjhi ${ }^{3}$, Mehedi Masud ${ }^{4}$ and Mohammed Baz \\ ${ }^{1}$ Department of Electronics and Communication Engineering, Raghu Institute of Technology (A), Visakhapatnam, \\ 531162, India \\ ${ }^{2}$ Department of Computer Science and Engineering, Chandigarh University, Mohali, 140413, India \\ ${ }^{3}$ School of Computer Science and Engineering, SCE, Taylor's University, Subang Jaya Malaysia \\ ${ }^{4}$ Department of Computer Science, College of Computers and Information Technology, Taif University, Taif, 21944, \\ Saudi Arabia \\ ${ }^{5}$ Department of Computer Engineering, College of Computer and Information Technology, Taif University, Taif, 21994, \\ Saudi Arabia \\ *Corresponding Author: Sahil Verma. Email: sahilverma@ieee.org \\ Received: 13 June 2021; Accepted: 24 September 2021
}

\begin{abstract}
Segmentation of vessel in retinal fundus images is a primary step for the clinical identification for specific eye diseases. Effective diagnosis of vascular pathologies from angiographic images is thus a vital aspect and generally depends on segmentation of vascular structure. Although various approaches for retinal vessel segmentation are extensively utilized, however, the responses are lower at vessel's edges. The curvelet transform signifies edges better than wavelets, and hence convenient for multiscale edge enhancement. The bilateral filter is a nonlinear filter that is capable of providing effective smoothing while preserving strong edges. Fast bilateral filter is an advanced version of bilateral filter that regulates the contrast while preserving the edges. Therefore, in this paper a fusion algorithm is recommended by fusing fast bilateral filter that can effectively preserve the edge details and curvelet transform that has better capability to detect the edge direction feature and better investigation and tracking of significant characteristics of the image. Afterwards $\mathrm{C}$ mean thresholding is used for the extraction of vessel. The recommended fusion approach is assessed on DRIVE dataset. Experimental results illustrate that the fusion algorithm preserved the advantages of the both and provides better result. The results demonstrate that the recommended method outperforms the traditional approaches.
\end{abstract}

Keywords: Blood vessel extraction; curvelet transform; fast bilateral filter; C mean thresholding

\section{Introduction}

Blood vessels are the preeminent and utmost steady structure that gives the idea inside the retina that can be directly inspected in vivo. The success of analysis for ophthalmologic ailments is relying 
on the timely recognition and modification in pathology of retina. The physical labelling of blood vessels is a tedious procedure which requires expert trainer. Automated segmentation offers steadiness and accurateness and decreases the time consumption by a surgeon or a technical expert for manual portraying. Thus, an automatic certain technique of vessel segmentation is beneficial for the rapid identification and characterization of the morphological variations in the retinal blood vessel. Usually, the automated extraction of the retinal images is a difficult task. The leading complications in the retina images are the insufficient contrast, illumination differences, noise effect, and anatomic changeability subjected to the individual patient. The treelike geometry is frequently twisted and complex due to which the features such as bifurcations and overlaps may mislead the recognition scheme. Furthermore, the challenges encounter in automated vessel recognition involves a broad variety of vessel widths, low contrast over background, and presence of various of structures in the image comprising of optic disc, the retinal boundary, the lesions, and other pathologies.

Numerous principles and approaches for the segmentation of retinal blood vessel are described in the literature. Fraz et al. [1] have given a detailed report for the various approaches available for the retinal vessel segmentation. Detection of retinal blood vessel segmentation is classified into techniques on the basis of pattern recognition, match filtering technique, morphological approach, vessel tracking, multiscale analysis, and model-based algorithms. The pattern recognition approaches are categorized into two types: supervised approaches and unsupervised approaches. Etraction of blood vessel features and classification is coming under supervised approach. These approaches comprise principal component analysis, neural networks, $\mathrm{k}$ nearest neighbour classifiers, support vector machine (SVM). Some of the unsupervised approaches that use ground truth data include matched filtering along with specially weighted fuzzy $\mathrm{C}$-means clustering, radius based clustering algorithm, maximum likelihood estimation of vessel parameters.

In spite of the fact that continuous development and efforts are addressed in the area of fundus image analysis, various challenges still required to be overcome. Furthermore, noise and low contrast still express as a vital hindrance to accomplish the high-quality enhancement, especially for optical imaging.

Thus, this work recommends a new algorithm for the enhancement of the vasculature by fusing traditional curvelet transform with fast bilateral filter (FBF) and top hat filter. In this work multiwavelet transformation is explored by utilizing curvelet transform that provides superior spatial and spectral localization of retinal image in comparison to other multi-scale representations. The reason is that the curvelet transform handles curve discontinuities efficiently with small number of coefficients. For denoising and preserving the edges of the retinal images FBF is used that consists of range and space filter. For space filtering, values nominated demonstrate the preferred amount of combination of pixels, while the range filtering selects values based on the low pass filtering. The FBF technique requires two parameters: range parameter $\left(\sigma_{r}\right)$ and spatial parameter $\left(\sigma_{s}\right)$ that control the response of filter. Therefore, it is highly necessary to select the values of the two parameters carefully to achieve better accuracy possible. In the next step top hat transform is applied to highlight the vasculature against the background. For segmentation of retinal blood vessel C-mean thresholding is employed.

The execution and strength of the suggested technique is verified on DRIVE retinal image database. The results obtained in this work shows remarkable achievement, almost close to the stateof-art approaches recently available in the literature. In comparison to other studies, the suggested approach has numerous superiorities, such as the edges of the retinal images can be enhanced by directly modifying the curvelet coefficients, and simultaneously edges can be preserved during the denoising process through FBF. 
Some of the relevant works related to supervised and unsupervised approaches for retinal vessel segmentation and bilateral filtering used for various applications that have been published are discussed below.

\section{Related Work}

Generally, most of the vessel segmentation approaches take up the green component of the image, since the noise level is lower and contrast is higher in this channel. Soares et al. [2] have recommended an approach, which grouped the pixels as vessel or non-vessel once utilizing supervised classification. Lupascu et al. [3] have utilized AdaBoost for the construction of a classifier. Chaudhuri et al. [4] Have proposed approaches that depends on matched filtering convolve with 2-Dimensional (2D) templates and are configured to represent the features of the vasculature. Kovacs et al. [5] also suggested an approach depend on matching of template and contour reconstruction. Annunziata et al. [6] have recommended a method in which the presence of exudates in retinal images are reported. Dashtbozorg et al. [7] have suggested a new approach to classify the blood vessels that depends on geometrical structure of vessels. Estrada et al. [8] have proposed a graph theoretical method by extending a global likelihood technique. Relan et al. [9] have employed least square-support vector machine approach for the classification of veins on four-color features. Vascular tortuosity measurement is vital for diagnosing of diabetes and several diseases related to central nervous system. Hart et al. [10] have suggested a tortuosity measurement and classification of vessel segmentation and networks, also summarized the previous works. Grisan et al. [11] have recommended a new technique to evaluate the tortuosity through partitioned of every segmented vessel and afterwards combined every evaluation. Wang et al. [12] have recommended a multiwavelet kernels and multiscale hierarchical decomposition for vessel segmentation. Fathi et al. [13] have recommended a method to segment the vessel and estimate the diameter of the vessel using automatic wavelet transform. Aslani et al. [14] have suggested a supervised technique based on robust hybrid features for the segmentation of vessel. Azzopardi et al. [15] have recommended COSFIRE filters for the segmentation of vessel. Roychowdhury et al. [16] have recommended extraction of major vessel and classification of subimage to segment the blood vessels. Roychowdhury et al. [17] have recommended an iterative vessel segmentation. Imani et al. [18] have proposed a technique for vessel identification through morphological component analysis. Panda et al. [19] have done the vessel segmentation through Binary Hausdorff Symmetry measure using growing of Seeded region. Tan et al. [20] have extracted the vessel using salient points network. Rodrigues et al. [21] have recommended segmenting the blood vessels and optic disc utilizing wavelets, morphology, and Hessian-based multiscale filtering. Farokhian et al. [22] have recommended segmenting of retinal vessel utilizing automatic parameters selection of gabor filter. Jiang et al. [23] have recommended an approach of segmenting the blood vessel utilizing fully convolutional network with transfer learning. Wang et al. [24] have proposed s cascade classification technique to segment the blood vessel. Sazak et al. [25] have recommended a vessel enhancement and extraction method using multiscale bowler-hat transform. Primitivo et al. [26] have suggested a hybrid model by combining Lateral Inhibition and Differential Evolution for retinal vessel segmentation. Shah et al. [27] have recommended a model of Gabor wavelet and line detector for vessel extraction. Dash and Senapati have enhanced the detection of vessel using a fusion of discrete wavelet transform (DWT) with Gamma Corrections and coye filter [28-30].

Multiresolution analysis has been effectively utilized in image processing particularly in segmenting the image. In recent times, the finite ridgelet and curvelet transforms have been presented as a higher dimensional tool. Curvelet transform is an addition of wavelet transform that focuses to carry out exceptional phenomena arising alongside curved edges in $2 \mathrm{D}$ images. The generations of curvelet 
transforms are: i) First generation curvelet transform ("Continuous Curvelet Transform") and ii) Second generation Fast Discrete Curvelet Transform (FDCT). The decomposition of curvelet occurs in four steps functioning as smooth portioning, subband decomposition, analysing of Ridgelet and renormalization [31].

Many efforts have been introduced using curvelet transform in order to segment retinal images. In 2016, Aghamohamadian-Sharbaf et al. [32] have utilized curvlet transform for automatic classifying blood vessel tortuosity of retina. In 2011, Miri et al. [33] have recommended a new methodology of segmenting the retinal blood vessel utilizing multistructure morphology operators. Curvelet transform is employed for achieving multistructure morphology. Esmaeili et al. [34] have recommended a new technique for enhancing the retinal blood vessels using curvelet transform.

Even though enhancement of retinal blood vessel is one of the vital issues in segmentation, yet preserving of edges while denoising is also equally important matter that has to be considered during segmentation. Bilateral filtering is an approach through which the edges can be preserved while denoising the images. The main purpose of the bilateral filter is to replace the intensity of every pixel of the image with a weighted average of intensity values of close by pixels. Tomasi et al. [35] have initially suggested the edge-preserving filter named as bilateral filter. The fundamental idea of bilateral filtering is that it creates a nonlinear combination of similar pixel values. Afterwards there are many extensions of bilateral filter and implemented for various applications like illumination correction, dynamic range compression, photograph enhancement, multiresolution bilateral filtering for image denoising, fast estimation of bilateral filter utilizing signal processing method, and texture analysis [36,37]. Few authors have proposed an improvement in structure preservation by clearly counting the structure with a supplementary weight that depends on the local shape and orientation of the data for medical image representation [38,39].

The arrangement of the paper is as follows: In Section 2 brief review of the preliminary concepts are presented. In Section 3 "Proposed Methodology" the detail technique for retinal vessel extraction is explained. The results are discussed in In Section 4 "Experimental Evaluation". Lastly, some conclusions are drawn in Section 5 "Conclusions".

\section{Preliminary Concepts}

This section presents the significant aspects that are utilized in the proposed methodology are revised. They comprise curvlet transform, bilateral filter, fast bilateral filter, and top-hat transform.

\subsection{Curvelet Transform}

Candes et al. have suggested Curvelet transform that is derived from Ridgelet transform. The curvelet transform is appropriate for the image that is eradicated from discontinuities to the other side of curves. Curvelet transform handles curve discontinuities in a fine manner because it is designed to handle curves utilizing only a small number of coefficients. The multiwavelet transformation offers better spatial and spectral localization of image when compared with other multiscale representations. However, here the curvelets via wrapping is implemented as it is faster and have less computational complexity. In this technique, the Fourier plane is split into different concentric circles, mentioned as scale; individually these concentric circles are once more split into different angular divisions, mentioned as the orientation. This fusion of the scale and the angular division is notable as parabolic wedges. In the frequency domain the structural activity is captured by radial wedges, and high anisotropy and directional sensitivity are the integral features of the curvelet transform. For finding 
the curvelet coefficients, inverse FFT is computed on each scale and angle. The curvelet transform consists of four stages and implemented as given below.

Initially in the subband decomposition the image is first decomposed into $\log _{2} N$ ( $\mathrm{N}$ is the size of the image) wavelet subbands and then curvelet subbands are generated by forming partial reconstruction from these wavelet subbands at various levels. The subband decompositions denoted as

$f \rightarrow\left(P_{0} f, \Delta_{1} f, \Delta_{2} f, \ldots\right)$

where $P_{0} \rightarrow$ lowpass filter, $\Delta$ bandpass (highpass) filters

The image is distributed into resolution layers $P_{0}$. All layers include the particulars of various frequencies.

In the next step of smooth portioning, every subband is smoothly windowed into 'squares' of a suitable measure. A grid of dyadic squares is described as:

$I_{\left(s, k_{1}, k_{2}\right)}=\left[\frac{k_{1}}{2^{s}}, \frac{k_{1}+1}{2^{s}}\right] \times\left[\frac{k_{2}}{2^{s}}, \frac{k_{2}+1}{2^{s}}\right] \in I_{s}$

Let $\mathrm{P}$ be a smooth window function. For every square, $\mathrm{P}_{I}$ is a displacement of $P$ localized close to $I$. By the Multiplication of $\Delta_{s} f$ with $\mathrm{P}_{I}$ yields a smooth dissection of the function into 'squares'.

$h_{I}=\mathrm{P}_{I} \Delta_{s} f$

This stage follows the windowing partition of the subbands isolated in the former step of the algorithm.

$\Delta_{s} f=P_{I} \Delta_{s} f \quad I \in I_{s}$

In the next step of renormalization, every resultant square is renormalized to unit scale. For a dyadic square $\mathrm{Q}$, renormalized unit scale is given as below.

$g_{Q}=T_{Q}^{-1} h_{Q}$

Lastly, inverse curvelet transform is applied to achieve the curvelet enhanced image.

The digital curvelet transform applied on a 2D image $f(x, y)$, such that $0<x \leq M$ and $0<y \leq N$, gives a set of curvelet coefficients $\mathrm{C}\left(\mathrm{s}, \theta, k_{1}, k_{2}\right)$ as follows.

$C\left(s, \theta, k_{1} k_{2}\right)=\sum_{0<y \leq N}^{0<x \leq M} f(x, y) \varphi_{s, \theta, k_{1} k_{2}}(x, y)$

Here's' represents the scale or no of decomposition level, ' $\theta$ ' represents orientation, ' $\boldsymbol{k}_{1}$ ' and ' $\boldsymbol{k}_{2}$ ' indicate spatial location of curvelet, $\varphi$ and ' $f(x, y)$ ' indicates the image in spatial domain. Thinner and sharper curvelets can be obtained by increasing the decomposition levels. The schematic diagram of the general steps of the curvelet transform is given in Fig. 1. 
CMC, 2022, vol.71, no.2

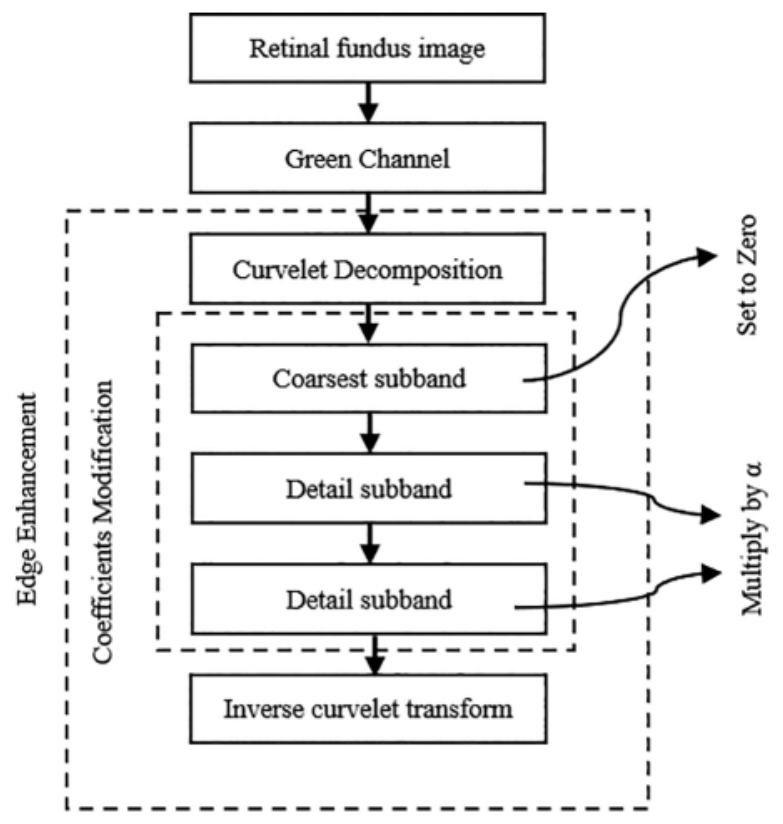

Figure 1: General steps of curvelet transform

\subsection{Bilateral Filter and Fast Bilateral Filter}

One of the vital issues of image processing is to successfully eliminate noise from an image while preserving its features. Noise elimination is a problematic assignment because various kinds of noises like additive, impulse or signal dependent noise may corrupt images. The solution is subjected to the nature of noise added to the image. The bilateral filter has better effects in eliminating noise while stabilizing edges in images. Afterwards many extensions of bilateral filter are done according to the requirement and to achieve better performance. One of the extensions is fast bilateral filter.

A standard form of the bilateral filter is considered in which a Gaussian kernel is utilized for range filtering, and a box or Gaussian kernel is utilized for spatial filtering. In this background, the bilateral filtering of an image $\{f(1): 1 \in I\}$, where $I$ is some finite rectangular domain of $Z^{2}$, is given as below.

$f_{B F}(1)=\frac{\sum_{j \in \Omega} w(j) g_{\sigma_{r}(f(1-j)-f(1)) f(1-j)}}{\sum_{j \in \Omega} w(j) g_{\sigma_{r}(f(1-j)-f(1))}}$

where

$g_{\sigma_{r}(t)}=\exp \left(-\frac{t^{2}}{2 \sigma_{r}^{2}}\right)$

The spatial filter is a Gaussian:

$w(1)=\exp \left(-\frac{\|1\|^{2}}{2 \sigma_{s}^{2}}\right)(1 \in \Omega)$

The fundamental background is to approximate directly the translated Gaussians appearing in Eq. (7) instead of approximating Eq. (8) and then translating the approximation in range space. 


\subsection{Top Hat Transform}

In image processing, top-hat transform is a process in which minute features and particulars are extracted from a specified image. Generally top-hat transforms are available in two different types such as white top-hat transform and black top-hat transform. The difference between the input image and its opening through some structuring element is known as white top-hat transform. Top-hat transforms are utilized for different image processing assignments like image enhancement, extraction of features, equalization of background etc. In this work white top-hat transform is utilized for retinal blood vessel enhancement. The white top-hat transform yields an image, comprising those elements of an input image which are brighter than its surroundings and smaller than the structuring element. Top-hat transformed images consist only non-negative values at all pixels. Let $P$ is the grayscale image and $s(x)$ be a grayscale structuring element then white Top-hat transform of $P$ is represented as follow.

$M(P)_{\text {tophat }}=P-(P o s)$

\section{Materials and Methods}

In this section, the suggested approach that combines top-hat transform and FBF with curvelet transform based on mean-C thresholding is suggested for vessel segmentation is explained in detail. The entire process comprises different operations and the entire proposal is split into three computing stages: preprocessing, processing, and post processing. Preprocessing stage consists of all the denoising and enhancement techniques. $\mathrm{C}$ mean thresholding is employed for segmentation in processing stage. Morphological cleaning is done in postprocessing stage.

The suggested approach contains of various steps. Initially, FBF is applied on retinal images. Further the filtered images are passed through curvelet-transformed. In the next step for highlighting the blood vessels against background top hat filter is applied. Mean-C thresholding is applied for the extraction of retinal blood vessel. The images acquired from the above process contain of some nonvessel that is eradicated with the help of morphological cleaning operation. A diagrammatic outline of the suggested method is narrated in Fig. 2 accompanied by output images.

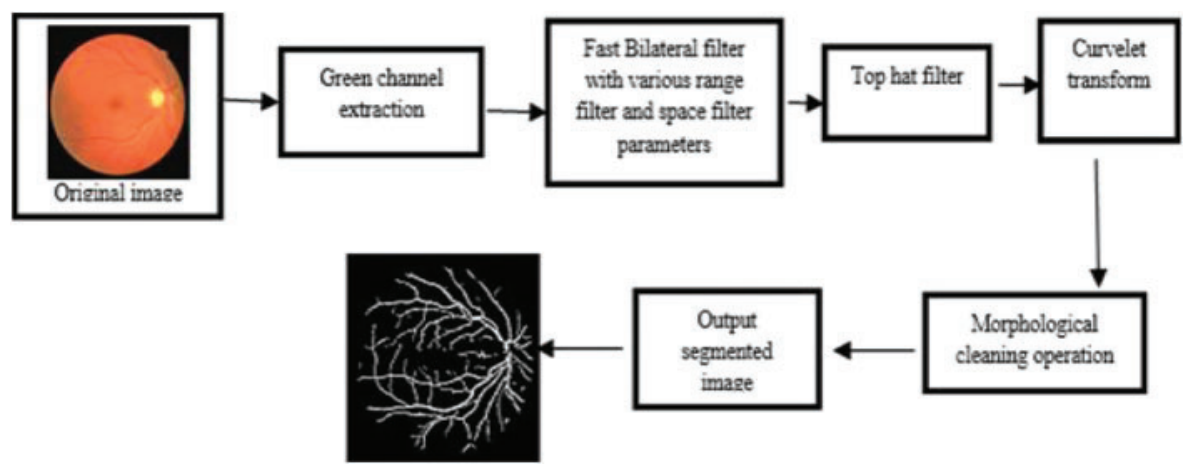

Figure 2: Schematic outline of the suggested methodology

To achieve superior performance accuracy, it is vital to work effectively on pre-processing stage. In this stage, few processes such as image enhancement, noise removal, removal of uneven background illumination are carried out. Thus, in retinal segmentation process pre-processing is a vital step. For the entire process of retinal vessel extraction, the green channel of the RGB image is chosen as it exhibits 
best contrast. Fig. 3a represents the original input image and Fig. $3 \mathrm{~b}$ represents the green channel image. Therefore, the steps of the suggested model start with extracting the green channel from the colour retina image. The proposed method comprises of various steps that are described as below.

(a)

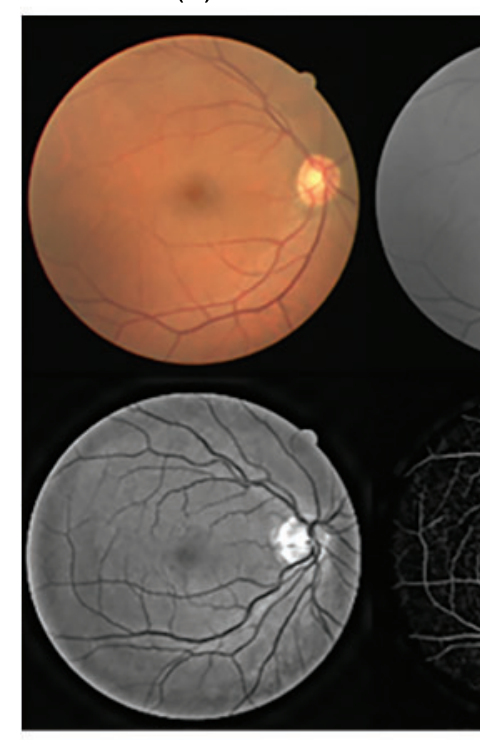

(d) (b)

(c)

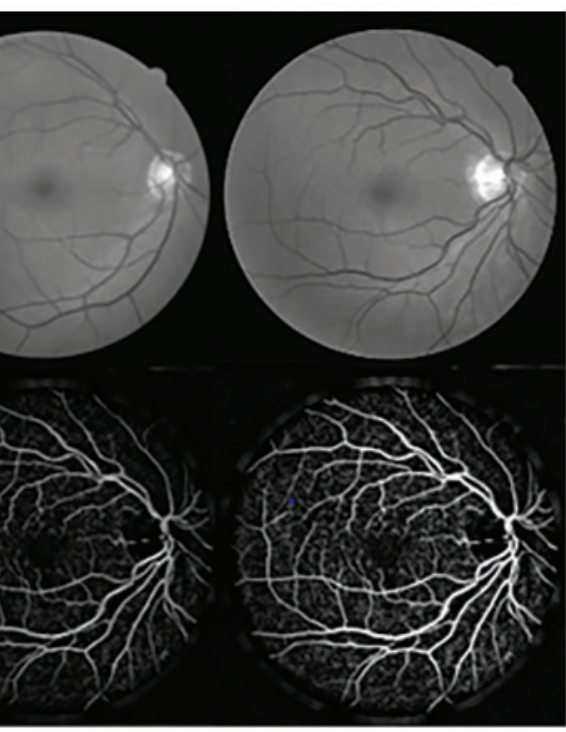

(e)

(f)

Figure 3: (a) Original retinal image (b) image extracted from green channel (c) fast bilateral filter transformed image (d) curvelet transform image (e) fast bilateral filter and curvelet transformed image (f) fast bilateral filter, curvelet, and top hat transformed image

\subsection{Preprocessing}

\subsubsection{Edge Preserving and Noise Removal by Using Fast Bilateral Filter}

In general, white Gaussian noise occurs in retinal images that has to be removed carefully and effectively without losing image information details. Thus, while filtering process retaining of the thin retinal vessels are challenging task. For noise removal FBF is utilized in the work. follows.

In general, the bilateral filter has numerous potentials that describe its accomplishment as

- It is simple to construct. Every pixel is substituted by a weighted average of its neighboring pixels. This characteristic is vital as it produces an uncomplicated filter to achieve its insight performance. Also, it helps to adjust and implement the filter according to the applicationspecific requirements.

- For preservation the size and contrast of the features are the two important parameters through which the bilateral filter is characterized

- It can be utilized in a non-iterative mode. This helps the parameters simple to fix because their consequence is not cumulative over various repetitions.

Hence, the two parameters that control the bilateral filter are range parameter $\left(\sigma_{r}\right)$ and spatial parameter $\left(\sigma_{s}\right)$. Also, the filter depends on window size. Parameters $\sigma_{r}$ and $\sigma_{s}$ define the amount of filtering for the input image. Even though bilateral filter is being utilized extensively, however, there 
is no substantial theoretical basis on selecting the optimum values. These values are often chosen by trial and error. Thus, in this work it is empirically analyzed and selected these parameters for image denoising.

Then bilateral filter is applied on the original retinal images by selecting different values of $\sigma_{r}$ and $\sigma_{s}$. For a fixed value of $\sigma_{s}$, retinal bilateral filtered images are generated with different values of $\sigma_{r}$. The window size of the bilateral filter is another important parameter and, in our method, we set it to be $5 \times 5$. The underlying idea is that images can be processed considering various values of $\sigma_{s}$ and $\sigma_{r}$, according to which the top hat transformed are derived into set of new images. These set of new images are further processed using curvelet transform and morphological cleaning operation, and to observe that at which combination of $\sigma_{s}$ and $\sigma_{r}$ enhanced retinal images are better preserved. Fig. 3c represents the FBF transformed image.

\subsubsection{Enhancement of Vasculature by Curvelet Transform}

The thicknesses of the retinal images slowly decrease when distance from optic disk is increased. The thick vessels fragmented into several thin branches. The illumination of the non-vessel regions is also decreased as the distance from the optic disk increased.

To overcome this challenge FBF transformed images are further processed through curvelet transform. The purpose of choosing curvelet transform is explained below.

In the curvelet transform, the curvelets are designed to pick up curves utilizing only a small number of coefficients. Therefore, the curve discontinuities are managed finely with curvelets. Main advantages of curvelet transform are its sensitivity towards directional edges and contours and its ability of representing them by less numbers of sparse nonzero coefficients. Thus, compared with wavelet transform, curvelet transform can effectively present the edges and curves with slighter number of coefficients. Furthermore, curvelet transforms is utilized to enhance the contrast of an image by highlighting its edges in several scales and directions. Fig. $3 \mathrm{~d}$ represents curvelet transformed image and Fig. 3e illustrates the fusion of FBF and curvelet transformed image.

\subsubsection{Highlighting of Vasculature by Top-Hat Filter}

Generally, the blood vessels are darker than surrounding tissues. To highlight the vessels top hat filter is employed. The above transformed images are further processed through top hat filter. The reason for selecting the top hat filter is described below.

In mathematical morphology, top-hat transform is a process of extraction of small or narrow, bright or dark features in an image. It is beneficial when variations in the background mean that a simple threshold cannot achieve this. Fig. 3f represents the final output image obtained from the fusion of FBF, curvelet transform, and top hat filter.

\subsection{Processing}

\subsubsection{Mean-C Thresholding for Vessel Extraction}

In this research mean-C thresholding method is considered. In this process based on local statistics like mean and median of the image thresholding is computed for every pixel. The threshold is upgraded every time. The core benefit of this approach is that it can be applied to uneven illuminated images. The steps for the mean-C thresholding are described as follows.

i. Initially the mean filter with window size $\mathrm{M} \times \mathrm{M}$ is chosen.

ii. The transformed image achieved through all the processes is convolved with mean. 
iii. By taking the difference of convolved image and transformed image, a new difference image is obtained.

iv. Considering a constant value $\mathrm{C}$, the difference image is thresholded.

v. The complement of thresholded image is computed.

\subsection{Postprocessing}

Once the vessel is extracted by applying thresholding, a postprocessing stage is applied for the elimination of noise or artifacts produced throughout the thresholding procedure. In this step, morphological cleaning operations: closing and opening are utilized to remove the non-vessel. One more essential cause of such process is to reconstruct those elements that are taken into consideration as a portion of vessel.

\section{Results and Discussion}

This section illustrates the efficiency of the recommended methodology when assessed over one publicly available DRIVE data image. This dataset is consisting of 40 colour fundus images of sizes $565 \times 584$ pixels 8 bits per colour channel and are taken by Canon CR 5 non mydriatic 3CCD camera with 458 field of view. It is divided into two sets of test and train both carrying 20 images. The training group images are physically segmented once, while the testing images are two times. Three human observers who are trained by an ophthalmologist are segmenting manually each retinal image. The resultant sets from manual segmentation of the test case are utilized as ground truth image for this work.

The efficacy of the recommended method is assessed by calculating different performance metrics like sensitivity (Sen), accuracy (Acc) and specificity (Spec) with different wavelets and different values of range and spatial parameters. Sensitivity quantifies the techniques of ability to detect the vessel pixel correctly while specificity is the computation of ability of the segmentation approach to mark non-vessel pixels. Accuracy is the computation of ability to find out the degree of conformity of the segmented image to the ground truth image.

For the computation of the performance of algorithm of the suggested approach, comparison of output of the segmented image and ground truth image is done by calculating the four parameters like true positive (TP), False negative (FN), true negative (TN), and false positive (FP). To analyse and quantify the method's efficiency the segmented result is compared with the ground truth and several performance measures like Sen, Acc and Spec are calculated.

Accuracy $=(T P+T N) /(T P+F N+T N+F P)$

Sensitivity $=T P /(T P+F N)$

Specificity $=T N /(T N+F N)$

Initially the performance metrics are evaluated for the original curvelet transform and the results are listed in Tab. 1. The performance metrics are computed for each image using the formulas given above and then the values are averaged in order to achieve a single performance measure. The Sen, Spec, and Acc attained for the original curvelet transform are $0.6537,0.9878$, and 0.9588 respectively. 
CMC, 2022, vol.71, no.2

2469

Table 1: Performance evaluation of original curvelet transform

\begin{tabular}{llll}
\hline Image & Sen & Spec & Acc \\
\hline R1 & 0.698404 & 0.987735 & 0.96192 \\
R2 & 0.67813 & 0.991474 & 0.95938 \\
R3 & 0.61317 & 0.986996 & 0.94973 \\
R4 & 0.623048 & 0.994029 & 0.9599 \\
R5 & 0.612157 & 0.993951 & 0.958183 \\
R6 & 0.591294 & 0.991724 & 0.95274 \\
R7 & 0.628814 & 0.986418 & 0.95374 \\
R8 & 0.611857 & 0.985877 & 0.953697 \\
R9 & 0.628398 & 0.989931 & 0.960632 \\
R10 & 0.621189 & 0.991681 & 0.961189 \\
R11 & 0.650259 & 0.986402 & 0.95631 \\
R12 & 0.655002 & 0.98716 & 0.95848 \\
R13 & 0.582163 & 0.982489 & 0.952373 \\
R14 & 0.707126 & 0.980249 & 0.958168 \\
R15 & 0.701194 & 0.980372 & 0.960392 \\
R16 & 0.669967 & 0.989716 & 0.960847 \\
R17 & 0.653239 & 0.986442 & 0.958316 \\
R18 & 0.685932 & 0.986232 & 0.962438 \\
R19 & 0.780461 & 0.991064 & 0.973594 \\
R20 & 0.682629 & 0.988011 & 0.965553 \\
Average & 0.6537 & 0.9878 & 0.9588 \\
\hline
\end{tabular}

The next step is the suggested approach of fusion of FBF, curvelet transform, and top-hat filter in which performance metrics are evaluated by taking various values of $\sigma_{r}$ and $\sigma_{s}$. The various values chosen for $\sigma_{s}$ are $0.3,0.4$ and 0.5 , similarly the values selected for $\sigma_{r}$ are 5, 6, 7, 8, and 9. For a particular fixed value of $\sigma_{s}$ different values of $\sigma_{r}$ are applied to the retinal images, and afterwards the performance metrics achieved by following the steps as described in Section 4. The results obtained are tabulated in Tabs. 2-4 respectively. Even though, it is observed that for many different combinations of $\sigma_{s}$ and $\sigma_{r}$ incremented performance metrics are obtained, however, only the highest values are considered for the averaging of each performance measure and listed in the tables. When $\sigma_{s}=0.3$ from Tab. 2 the corresponding average Sen, Spec, and Acc achieved are 0.6791, 0.9899, and 0.9621. When $\sigma_{s}=0.4$ from Tab. 3 the corresponding average Sen, Spec, and Acc attained are 0.6813, 0.9900, and 0.9629. When $\sigma_{s}=0.5$ from Tab. 4 the corresponding average Sen, Spec, and Acc attained are 0.6907, 0.9904, and 0.9640 . Consequently, as the combination of $\sigma_{s}$ at 0.5 with various values of $\sigma_{r}$ delivers the best results and that are taken as final values for comparing with other approaches. 
Table 2: Performance evaluation curvelet transform based on FBF with $\sigma_{\mathrm{s}}=0.3$

\begin{tabular}{llll}
\hline $\begin{array}{l}\text { Retinal images } \\
\text { with various } \sigma_{r}\end{array}$ & Sen & Spec & Acc \\
\hline $\mathrm{R} 1, \sigma_{r}=7$ & 0.716912 & 0.988968 & 0.967783 \\
$\mathrm{R} 2, \sigma_{r}=6$ & 0.696388 & 0.993966 & 0.963492 \\
$\mathrm{R} 3, \sigma_{r}=6$ & 0.659465 & 0.989909 & 0.958576 \\
$\mathrm{R} 4, \sigma_{r}=9$ & 0.673177 & 0.996073 & 0.966977 \\
$\mathrm{R} 5, \sigma_{r}=7$ & 0.689076 & 0.99562 & 0.959749 \\
$\mathrm{R} 6, \sigma_{r}=6$ & 0.639327 & 0.991367 & 0.954403 \\
$\mathrm{R} 7, \sigma_{r}=5$ & 0.651864 & 0.988272 & 0.961079 \\
$\mathrm{R} 8, \sigma_{r}=5$ & 0.629395 & 0.986187 & 0.956973 \\
$\mathrm{R} 9, \sigma_{r}=8$ & 0.63771 & 0.98996 & 0.965207 \\
$\mathrm{R} 10, \sigma_{r}=8$ & 0.636397 & 0.990937 & 0.964922 \\
$\mathrm{R} 11, \sigma_{r}=7$ & 0.669352 & 0.988397 & 0.959282 \\
$\mathrm{R} 12, \sigma_{r}=6$ & 0.668059 & 0.989518 & 0.959828 \\
$\mathrm{R} 13, \sigma_{r}=9$ & 0.601104 & 0.990329 & 0.958276 \\
$\mathrm{R} 14, \sigma_{r}=7$ & 0.725306 & 0.983678 & 0.962252 \\
$\mathrm{R} 15, \sigma_{r}=8$ & 0.720124 & 0.982177 & 0.964852 \\
$\mathrm{R} 16, \sigma_{r}=6$ & 0.691954 & 0.990924 & 0.96302 \\
$\mathrm{R} 17, \sigma_{r}=8$ & 0.653526 & 0.988721 & 0.953849 \\
$\mathrm{R} 18, \sigma_{r}=8$ & 0.705421 & 0.988836 & 0.963347 \\
$\mathrm{R} 19 \sigma_{r}=5$ & 0.806949 & 0.993701 & 0.974357 \\
$\mathrm{R} 20, \sigma_{r}=8$ & 0.712122 & 0.992293 & 0.964468 \\
\hline
\end{tabular}

Table 3: Performance evaluation curvelet transform based on FBF with $\sigma_{s}=0.4$

\begin{tabular}{llll}
\hline $\begin{array}{l}\text { Retinal images } \\
\text { with various } \sigma_{r}\end{array}$ & Sen & Spec & Acc \\
\hline $\mathrm{R} 1, \sigma_{r}=6$ & 0.716644 & 0.987845 & 0.962826 \\
$\mathrm{R} 2, \sigma_{r}=8$ & 0.692956 & 0.993783 & 0.960489 \\
$\mathrm{R} 3, \sigma_{r}=7$ & 0.661046 & 0.988893 & 0.960718 \\
$\mathrm{R} 4, \sigma_{r}=8$ & 0.684198 & 0.995003 & 0.969807 \\
$\mathrm{R} 5, \sigma_{r}=5$ & 0.679723 & 0.994774 & 0.959949 \\
$\mathrm{R} 6, \sigma_{r}=8$ & 0.643873 & 0.992102 & 0.958606 \\
$\mathrm{R} 7, \sigma_{r}=5$ & 0.654295 & 0.991542 & 0.964364 \\
$\mathrm{R} 8, \sigma_{r}=7$ & 0.622706 & 0.98758 & 0.959703 \\
$\mathrm{R} 9, \sigma_{r}=6$ & 0.639206 & 0.990062 & 0.966871 \\
$\mathrm{R} 10, \sigma_{r}=8$ & 0.638607 & 0.994013 & 0.962994 \\
$\mathrm{R} 11, \sigma_{r}=8$ & 0.671756 & 0.988144 & 0.960267 \\
\hline
\end{tabular}

(Continued) 
Table 3: Continued

\begin{tabular}{llll}
\hline $\begin{array}{l}\text { Retinal images } \\
\text { with various } \sigma_{r}\end{array}$ & Sen & Spec & Acc \\
\hline $\mathrm{R} 12, \sigma_{r}=8$ & 0.671183 & 0.988806 & 0.959913 \\
$\mathrm{R} 13, \sigma_{r}=9$ & 0.614265 & 0.990889 & 0.956188 \\
$\mathrm{R} 14, \sigma_{r}=8$ & 0.72898 & 0.984971 & 0.959082 \\
$\mathrm{R} 15, \sigma_{r}=6$ & 0.728775 & 0.983772 & 0.961595 \\
$\mathrm{R} 16, \sigma_{r}=6$ & 0.698196 & 0.99062 & 0.962038 \\
$\mathrm{R} 17, \sigma_{r}=7$ & 0.659721 & 0.986287 & 0.959722 \\
$\mathrm{R} 18, \sigma_{r}=9$ & 0.708751 & 0.987595 & 0.964501 \\
$\mathrm{R} 19, \sigma_{r}=5$ & 0.807607 & 0.993444 & 0.976361 \\
$\mathrm{R} 20, \sigma_{r}=6$ & 0.703524 & 0.989211 & 0.966496 \\
\hline
\end{tabular}

Table 4: Performance evaluation curvelet transform based on FBF with $\sigma_{s}=0.5$

\begin{tabular}{llll}
\hline $\begin{array}{l}\text { Retinal images } \\
\text { with various } \sigma_{r}\end{array}$ & Sen & Spec & Acc \\
\hline $\mathrm{R} 1, \sigma_{r}=7$ & 0.724334 & 0.989294 & 0.963029 \\
$\mathrm{R} 2, \sigma_{r}=6$ & 0.709494 & 0.992209 & 0.965516 \\
$\mathrm{R} 3, \sigma_{r}=6$ & 0.667262 & 0.989913 & 0.950857 \\
$\mathrm{R} 4, \sigma_{r}=6$ & 0.688433 & 0.992837 & 0.968235 \\
$\mathrm{R} 5, \sigma_{r}=8$ & 0.689011 & 0.991747 & 0.962765 \\
$\mathrm{R} 6, \sigma_{r}=8$ & 0.657901 & 0.995639 & 0.958191 \\
$\mathrm{R} 7, \sigma_{r}=7$ & 0.657731 & 0.982292 & 0.958085 \\
$\mathrm{R} 8, \sigma_{r}=9$ & 0.638911 & 0.988344 & 0.958591 \\
$\mathrm{R} 9, \sigma_{r}=9$ & 0.65061 & 0.99197 & 0.965386 \\
$\mathrm{R} 10, \sigma_{r}=5$ & 0.65587 & 0.993571 & 0.968826 \\
$\mathrm{R} 11, \sigma_{r}=8$ & 0.685371 & 0.988059 & 0.962798 \\
$\mathrm{R} 12, \sigma_{r}=7$ & 0.683411 & 0.989383 & 0.963446 \\
$\mathrm{R} 13, \sigma_{r}=5$ & 0.625879 & 0.990498 & 0.959234 \\
$\mathrm{R} 14, \sigma_{r}=9$ & 0.735154 & 0.988864 & 0.96108 \\
$\mathrm{R} 15, \sigma_{r}=8$ & 0.731945 & 0.98817 & 0.968904 \\
$\mathrm{R} 16, \sigma_{r}=8$ & 0.699109 & 0.990747 & 0.968326 \\
$\mathrm{R} 17, \sigma_{r}=9$ & 0.667461 & 0.98986 & 0.963646 \\
$\mathrm{R} 18, \sigma_{r}=9$ & 0.714989 & 0.989803 & 0.968695 \\
$\mathrm{R} 19, \sigma_{r}=5$ & 0.814793 & 0.995815 & 0.977467 \\
$\mathrm{R} 20, \sigma_{r}=6$ & 0.713079 & 0.99077 & 0.968202 \\
\hline
\end{tabular}

Note: In all the tables R represents retina.

Several methodologies are suggested in the literature that comprise both supervised and unsupervised segmentation methods. Few of the state-of-the-art algorithms are considered to compare with the suggested technique. The execution of the recommended method on DRIVE dataset is compared with other methods correspondence to Sen, Spec, and Acc. Tab. 5 demonstrates the 
accomplishment of the recommended methodology with different supervised and unsupervised methods stated by Wang et al. [12], Fathi et al. [13], Azzopardi et al. [15], Roychowdhury et al. [16], Roychowdhury et al. [17], Imani et al. [18], Aslani et al. [14], Panda et al. [19], Tan et al. [20], Rodrigues et al. [21], Farokhian et al. [22], Jiang et al. [23], Sazak et al. [25], Primitivo et al. [26], Shah et al. [27], and Dash et al. [28] on DRIVE database.

Table 5: Performance measures comparison for various algorithms

\begin{tabular}{lllll}
\hline Year & Method & Sen & Spec & Acc \\
\hline 2013 & Wang et al. [12] & N.A & N.A & 0.9461 \\
2013 & Fathi et al. [13] & 0.7768 & 0.9759 & 0.9581 \\
2015 & Azzopardi et al. [15] & 0.765 & 0.97 & 0.944 \\
2015 & Roychowdhury et al. [16] & 0.725 & 0.983 & 0.952 \\
2015 & Roychowdhury et al. [17] & 0.739 & 0.978 & 0.949 \\
2015 & Imani et al. [18] & 0.7524 & 0.9753 & 0.9523 \\
2016 & Aslani et al. [14] & 0.7545 & 0.9801 & 0.9513 \\
2016 & Panda et al. [19] & 0.733 & 0.975 & 0.953 \\
2016 & Tan et al. [20] & N.A & N.A & 0.93 \\
2017 & Rodrigues et al. [21] & 0.7223 & 0.9636 & 0.9472 \\
2017 & Farokhian et al. [22] & 0.693 & 0.979 & 0.939 \\
2018 & Jiang et al. [23] & 0.7121 & 0.9832 & 0.9593 \\
2019 & Sazak et al. [25] & 0.718 & 0.981 & 0.959 \\
2019 & Primitivo et al. [26] & 0.8464 & 0.9701 & 0.9619 \\
2019 & Shah et al. [27] & 0.7421 & NA & 0.947 \\
2020 & Dash et al. [28] & 0.7403 & 0.9905 & 0.9661 \\
- & Traditional curvelet & 0.6537 & 0.9878 & 0.9588 \\
& transform & & & \\
- & Proposed method & 0.6907 & 0.9904 & 0.964 \\
& (FBF + curvelet + top hat) & & & \\
\hline
\end{tabular}

Note: N.A: not available.

Fig. 4 demonstrates the comparison bar graph of the recommended approach with original curvelet transform and other few suggested approaches with three performance measures such as sensitivity, specificity, and accuracy. In Fig. 4, OC represents original curvelet and PM represents proposed method and the number represents the reference number of the other suggested approaches.

The vessel extraction results by the suggested approach of three retinal images like retina 1,2 , and 4 for DRIVE database are presented in Fig. 4. The first column of Fig. 4 illustrates the original images. The second column denotes the ground truth images. The third column represents the vessel extraction results using curvelet transform approach. The fourth column shows the vessel extraction results using the suggested approach. Comparing the results presented in 3rd column with ground truth images, it is noticed that the original curvelet transform approach is unsuccessful for extraction of few tiny vessels and contain false elements as element of vessel. Fig. 5 displays the output segmented images obtained from the recommended approach on DRIVE dataset. 


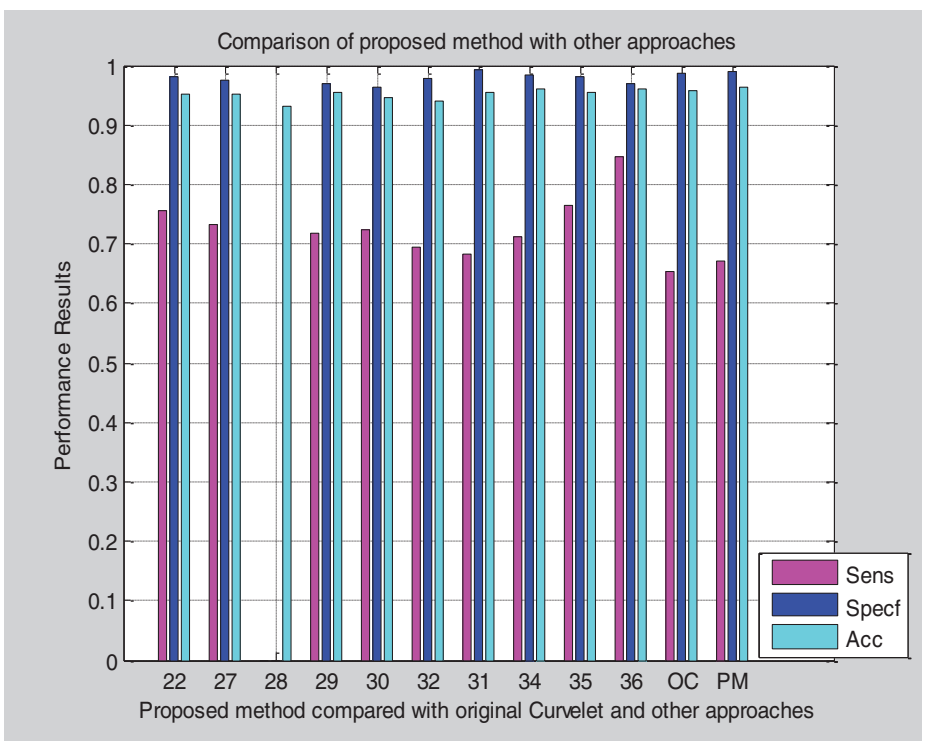

Figure 4: Comparison of proposed method with original curvelet and other approaches
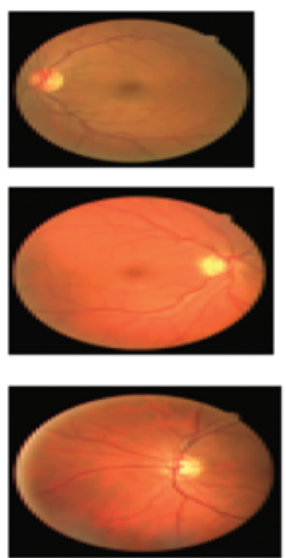

(a)
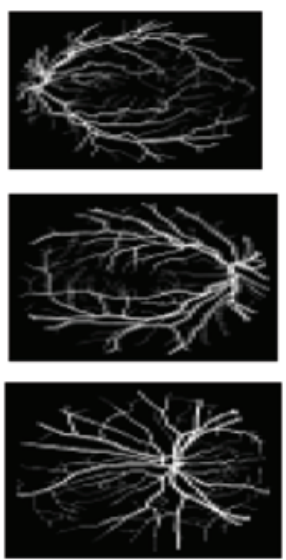

(b)
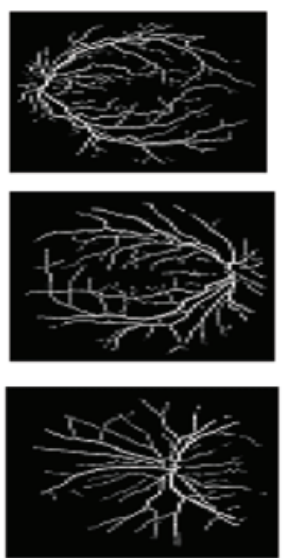

(c)
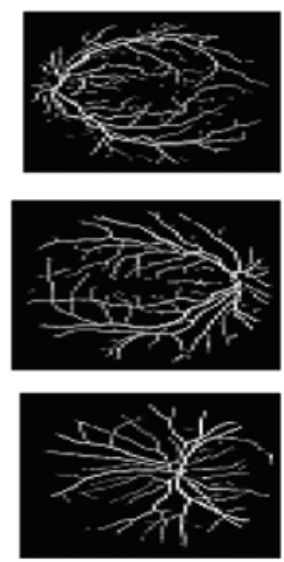

(d)

Figure 5: (a) Original retinal image (b) ground truth images (c) vessel extracted from median filter and curvelet transform (d) vessel extracted from fusion model of FBF, top hat and curvelet transform

The suggested approach, under the alike circumstances, outperforms in identifying the tiny vessels. It might have important to state that the suggested approaches are based on unsupervised technique that generally considers the training dataset.

\section{Conclusion}

In this work, a fusion model of FBF, curvelet transform, and top-hat filter techniques is presented. Vessels are extracted utilizing $\mathrm{C}$ mean thresholding. The important contribution of the paper is to enhance the performance of the original curvelet transform further by combining with different techniques for the analysis of retinal blood vessel. The recommended approach is assessed using DRIVE database. In order to signify the accomplishment of the recommended approach extensive 
simulation results of DRIVE dataset are presented and compared with different other approaches. The suggested approach achieves accuracy of 0.9640 that is higher than the original curvelet transform. The recommended method is highly effectual in identifying both the large and tiny vessels with high values of sensitivity and specificity 0.6907 and 0.9904 respectively. The constraint of the recommended approach is that in some retinal images it is incapable to retain the connection that can give on to imprecise segmentation outcomes.

Funding Statement: The authors would like to thank for the support from Taif University Researchers Supporting Project number (TURSP-2020/239), Taif University, Taif, Saudi Arabia.

Conflicts of Interest: The authors declare that they have no conflicts of interest to report regarding the present study.

\section{References}

[1] M. M. Fraz, P. Remagnino, A. Hoppe, B. Uyyanonvara, A. R. Rudnicka et al., "Blood vessel segmentation methodologies in retinal images - a survey," Computer Methods and Programs in Biomedicine, vol. 108, no. 1, pp. 407-433, 2012.

[2] J. V. B. Soares, J. J. G. Leandro, R. M. Cesar, H. F. Jelinek and M. J. Cree, "Retinal vessel segmentation using the 2-d gabor wavelet and supervised classification," IEEE Transactions on Medical Imaging, vol. 25, no. 9, pp. 1214-1222, 2006.

[3] C. A. Lupaşcu, D. Tegolo and E. Trucco, "FABC: Retinal vessel segmentation using adaBoost," IEEE Transactions on Information Technology in Biomedicine, vol. 14, no. 5, pp. 1267-1274, 2010.

[4] S. Chaudhuri, S. Chatterjee, N. Katz, M. Nelson and M. Goldbaum, "Detection of blood vessels in retinal images using two-dimensional matched filters," IEEE Transactions on Medical Imaging, vol. 8, no. 3, pp. 263-269, 1989.

[5] G. Kovács and A. Hajdu, "A self-calibrating approach for the segmentation of retinal vessels by template matching and contour reconstruction," Medical Image Analysis, vol. 29, pp. 24-46, 2016.

[6] R. Annunziata, A. Garzelli, L. Ballerini, A. Mecocci and E. Trucco, "Leveraging multiscale hessian-based enhancement with a novel exudate inpainting technique for retinal vessel segmentation," IEEE Journal of Biomedical and Health Informatics, vol. 20, no. 4, pp. 1129-1138, 2016.

[7] B. Dashtbozorg, A. M. Mendonca and A. Campilho, "An automatic graph-based approach for artery/Vein classification in retinal images," IEEE Transactions on Image Processing, vol. 23, no. 3, pp. 1073-1083, 2014.

[8] R. Estrada, M. J. Allingham, P. S. Mettu, S. W. Cousins, C. Tomasi et al., "Retinal artery-vein classification via topology estimation," IEEE Transactions on Medical Imaging, vol. 34, no. 12, pp. 2518-2534, 2015.

[9] D. Relan, T. MacGillivray, L. Ballerini, E. Trucco, "Automatic retinal vessel classification using a least square-support vector machine in VAMPIRE," in Proc. EMBC, Chicago, IL, USA, pp. 142-145, 2014.

[10] W. E. Hart, M. Goldbaum, B. Côté, P. Kube and M. R. Nelson, "Measurement and classification of retinal vascular tortuosity," International Journal of Medical Informatics, vol. 53, no. 2-3, pp. 239-252, 1999.

[11] E. Grisan, M. Foracchia and A. Ruggeri, "A novel method for the automatic grading of retinal vessel tortuosity," IEEE Transactions on Medical Imaging, vol. 27, no. 3, pp. 310-319, 2008.

[12] Y. Wang, G. Ji, P. Lin and E. Trucco, "Retinal vessel segmentation using multiwavelet kernels and multiscale hierarchical decomposition," Pattern Recognition, vol. 46, no. 8, pp. 2117-2133, 2013.

[13] A. Fathi and A. R. Naghsh-Nilchi, "Automatic wavelet-based retinal blood vessels segmentation and vessel diameter estimation," Biomedical Signal Processing and Control, vol. 8, no. 1, pp. 71-80, 2013.

[14] S. Aslani and H. Sarnel, "A new supervised retinal vessel segmentation method based on robust hybrid features," Biomedical Signal Processing and Control, vol. 30, pp. 1-12, 2016.

[15] G. Azzopardi, N. Strisciuglio, M. Vento and N. Petkov, "Trainable COSFIRE filters for vessel delineation with application to retinal images," Medical Image Analysis, vol. 19, no. 1, pp. 46-57, 2015. 
[16] S. Roychowdhury, D. D. Koozekanani and K. K. Parhi, "Blood vessel segmentation of fundus images by major vessel extraction and subimage classification," IEEE Journal of Biomedical and Health Informatics, vol. 19, no. 3, pp. 1118-1128, 2015.

[17] S. Roychowdhury, D. D. Koozekanani and K. K. Parhi, "Iterative vessel segmentation of fundus images," IEEE Transactions on Biomedical Engineering, vol. 62, no. 7, pp. 1738-1749, 2015.

[18] E. Imani, M. Javidi and H. R. Pourreza, "Improvement of retinal blood vessel detection using morphological component analysis," Computer Methods and Programs in Biomedicine, vol. 118, no. 3, pp. 263-279, 2015.

[19] R. Panda, N. B. Puhan and G. Panda, "New binary hausdorff symmetry measure based seeded region growing for retinal vessel segmentation," Biocybernetics and Biomedical Engineering, vol. 36, no. 1, pp. 119-129, 2016.

[20] J. H. Tan, U. R. Acharya, K. C. Chua, C. Cheng and A. Laude, "Automated extraction of retinal vasculature," Medical Physics, vol. 43, no. 5, pp. 2311-2322, 2016.

[21] L. C. Rodrigues and M. Marengoni, "Segmentation of optic disc and blood vessels in retinal images using wavelets, mathematical morphology and hessian-based multi-scale filtering," Biomedical Signal Processing and Control, vol. 36, pp. 39-49, 2017.

[22] F. Farokhian, C. Yang, H. Demirel, S. Wu and I. Beheshti, "Automatic parameters selection of gabor filters with the imperialism competitive algorithm with application to retinal vessel segmentation," Biocybernetics and Biomedical Engineering, vol. 37, no. 1, pp. 246-254, 2017.

[23] Z. Jiang, H. Zhang, Y. Wang and S. B. Ko, "Retinal blood vessel segmentation using fully convolutional network with transfer learning," Computerized Medical Imaging and Graphics, vol. 68, pp. 1-15, 2018.

[24] X. Wang, X. Jiang and J. Ren, "Blood vessel segmentation from fundus image by a cascade classification framework," Pattern Recognition, vol. 88, pp. 331-341, 2019.

[25] C. Sazak, C. J. Nelson and B. Obara, "The multiscale bowler-hat transform for blood vessel enhancement in retinal images," Pattern Recognition, vol. 88, pp. 739-750, 2019.

[26] D. Primitivo, R. Alma, C. Erik, V. Arturo, C. Edgar et al., "A hybrid method for blood vessel segmentation in images," Biocybernetics and Biomedical Engineering, vol. 39, no. 3, pp. 814-824, 2019.

[27] S. A. A. Shah, A. Shahzad, M. A. Khan, C. K. Lu and T. B. Tang, "Unsupervised method for retinal vessel segmentation based on gabor wavelet and multiscale line detector," IEEE Access, vol. 7, pp. 167221-167228, 2019.

[28] S. Dash and M. R. Senapati, "Enhancing detection of retinal blood vessels by combined approach of DWT, tyler coye and gamma correction," Biomedical Signal Processing and Control, vol. 57, pp. 1-12, 2020.

[29] I. Batra, S. Verma, Kavita, Uttam Ghosh, Joel J. P. C. Rodrigues et al., "Hybrid logical security framework for privacy preservation in the green internet of things," MDPI-Sustainability, vol. 12, no. 14, pp. 5542, 2020.

[30] B. Vijayalakshmi, K. Ramar, N. Z. Jhanjhi, S. Verma, M. Kaliappan et al., "An attention-based deep learning model for traffic flow prediction using spatiotemporal features towards sustainable smart city," International Journal Communication System, vol. 34, no. 3, 2021.

[31] E. Candès, L. Demanet, D. Donoho and L. Ying, "Fast discrete curvelet transforms," Multiscale Modeling and Simulation, vol. 5, no. 3, pp. 861-899, 2006.

[32] M. Aghamohamadian-Sharbaf, H. R. Pourreza and T. Banaee, "A novel curvature-based algorithm for automatic grading of retinal blood vessel tortuosity," IEEE Journal of Biomedical and Health Informatics, vol. 20, no. 2, pp. 586-595, 2016.

[33] M. S. Miri and A. Mahloojifar, "Retinal image analysis using curvelet transform and multistructure elements morphology by reconstruction," IEEE Transactions on Biomedical Engineering, vol. 58, no. 5, pp. 1183-1192, 2011.

[34] M. Esmaeili, H. Rabbani, A. Mehri and A. Dehghani, "Extraction of retinal blood vessels by curvelet transform," in Proc. ICIP, Cario, Egypt, pp. 3353-3356, 2009.

[35] C. Tomasi and R. Manduchi, "Bilateral filtering for gray and color images," in Proc. ICCV, Bombay, India, pp. 839-846, 1998. 
[36] M. Elad, "Retinex by two bilateral filters," in Lecture Notes in Computer Science, vol. 3459, pp. 217-229, 2005.

[37] F. Durand and J. Dorsey, "Fast bilateral filtering for the display of high-dynamic-range images," in $A C M$ Transactions on Graphics, vol. 21, no. 3, pp. 257-266, 2002.

[38] W. C. K. Wong, A. C. S. Chung and S. C. H. Yu, "Trilateral filtering for biomedical images," in 2004 2nd IEEE Int. Symp. on Biomedical Imaging: Nano to Macro, Arlington, VA, USA, vol. 1, pp. 820-823, 2004.

[39] W. Li, Y. Chai, F. Khan, S. R. U. Jan, S. Verma et al., "A comprehensive survey on machine learning-based Big data analytics for IoT-enabled smart healthcare system," Mobile Network and Applications, vol. 26, pp. 234-252, 2021. 